\title{
Alternative site testing
}

National Diabetes Information Clearinghouse (NDIC)

\section{Definitions}

Blood glucose monitoring

Defined by National Diabetes Information Clearinghouse (NDIC)

\section{Source}

National Diabetes Information Clearinghouse (U.S.). (2009). The diabetes dictionary. [Bethesda, Md.]: U.S. Dept. of Health and Human Services, National Institutes of Health, National Institute of Diabetes and Digestive and Kidney Diseases, National Diabetes Information Clearinghouse.

Using areas of the body other than the fingertips for blood glucose monitoring. 